\title{
Clinical characteristics of patients with leiomyoma who undergo surgery after high intensity focused ultrasound (HIFU)
}

\author{
Yun Seo Choe, Won Moo Lee, Joong Sub Choi, Jaeman Bae, Jeong Min Eom, Eunsaem Choi \\ Division of Gynecologic Oncology and Gynecologic Minimally Invasive Surgery, Department of Obstetrics and Gynecology, Hanyang University \\ College of Medicine, Seoul, Korea
}

\section{Objective}

We evaluated the clinical characteristics of patients who underwent surgery after high intensity focused ultrasound (HIFU) to treat uterine leiomyoma.

\section{Methods}

From June 2016 to September 2017, patients at our hospital who underwent HIFU to treat uterine leiomyoma prior to surgery were enrolled. All patients underwent pelvic magnetic resonance imaging (MRI) before and after HIFU. If 6 months had passed since the last pelvic MRI was performed, imaging was performed again before the operation.

\section{Results}

A total of 12 patients were analyzed. The median age was 45 (range, 28-51) years. The median body mass index was 24.9 (range, $18.1-29.2) \mathrm{kg} / \mathrm{m}^{2}$. The median size of the leiomyoma was 10.1 (range, 7.8-14.0) $\mathrm{cm}$ before HIFU, which changed to 8.75 (range, 5.9-14.8) cm after HIFU. The median size increased to 9.1 (range, 5.9-18.0) $\mathrm{cm}$ before the operation. Surgery was planned for several reasons, including an increase in the leiomyoma size $(n=6)$, persistent symptoms $(n=4)$, and newly developed lesion $(n=2)$. The median interval between HIFU and surgery was 7 (range, 3-32) months. Ten of the 12 patients underwent laparoscopic surgery, while the others underwent laparotomy; 6 patients also underwent laparoscopic myomectomy, and 4 underwent hysterectomy. Histopathologic findings showed infarction-type necrosis surrounded by granulation tissue with the infiltration of lymphocytes and macrophages in all patients.

\section{Conclusion}

Treatment of leiomyoma with operative procedures should be considered in selected patients with tumor size greater than $10 \mathrm{~cm}$, multiple tumors, and persistent symptoms after HIFU treatment.

Keywords: Leiomyoma; High-intensity focused ultrasound ablation; Myomectomy; Hysterectomy

\section{Introduction}

Leiomyoma is a monoclonal tumor that originates from smooth muscle cell proliferation and is the most common benign tumor of the uterus. Leiomyoma is frequently identified in women of childbearing age and has a reported incidence of $40-60 \%$ in 35 -year-olds, which increases to $70-80 \%$ in 50 -year-olds [1]. Leiomyoma is a hormone-responsive tumor that is dependent on sex hormones, such as estrogen and progesterone, and it can decrease or remain the same size after menopause [2]. Leiomyoma treatment is primar-
Received: 2018.09.22. Accepted: 2018.10.25.

Corresponding author: Won Moo Lee

Division of Gynecologic Oncology and Gynecologic Minimally Invasive Surgery, Department of Obstetrics and Gynecology, Hanyang University College of Medicine, 222 Wangsimni-ro Seongdong-gu, Seoul 04763, Korea

E-mail: leewmoo@hanyang.ac.kr

https://orcid.org/0000-0003-3433-9478

Articles published in Obstet Gynecol Sci are open-access, distributed under the terms of the Creative Commons Attribution Non-Commercial License (http://creativecommons. org/licenses/by-nc/3.0/) which permits unrestricted non-commercial use, distribution, and reproduction in any medium, provided the original work is properly cited.

Copyright (C) 2019 Korean Society of Obstetrics and Gynecology 


\section{Obstetrics \& Gynecology Science}

Yun Seo Choe, et al. Optimal condition of surgery after HIFU

ily based on the presence of symptoms, and regular followup is important. Patients who experience severe pelvic pain, massive vaginal bleeding, infertility, and pressure should undergo aggressive treatment. Additionally, a distinct increase in leiomyoma size during follow-up requires treatment $[2,3]$. Treatment approaches can vary and should take into consideration patient age, childbearing plans, and the location and size of the leiomyoma. Surgical management, medical therapy, uterine artery embolization, and myolysis can all be considered $[2,3]$.

High intensity focused ultrasound (HIFU) is widely used and advantageous as it is minimally invasive and does not cause injury to adjacent organs $[4,5]$. The mechanism of HIFU for tumor treatment is based on its heating effect, cavitation, and direct damage to tumor blood vessels, which results in coagulative necrosis of the targeted tissue $[4,5]$.

HIFU was previously applied as a noninvasive technique for treating hepatocellular carcinoma or prostate cancer [6] and was approved by the FDA in 2004 for uterine leiomyoma $[6,7]$. In Korea, HIFU was recognized as a new medical technology in 2009 and was typically used in patients wanting to preserve their uterus.

However, issues regarding the abuse of HIFU treatment and consequent complications were raised, and the Korean Society of Obstetrics and Gynecology (KSOG) published clinical practice guidelines in 2016 [8]. The guidelines state that patients with symptomatic uterine fibroids and premenopausal women aged over 18 years are indicated to undergo HIFU treatment [8]. Although further specific indications vary according to national and center guidelines, HIFU has been considered a viable option for women with no plans for future pregnancy, those who want to preserve the uterus, those with severe symptoms, and those with moderately sized leiomyoma without malignancy [9].

Unfortunately, there is little information regarding the indications for surgery or re-intervention after HIFU failure. In a recent study, the re-intervention risk after 60 months was reported to be $53.9 \%$ (95\% confidence interval, $47.2-60.4 \%$ ) for HIFU; however, they did not definitively clarify the indications for the re-intervention after HIFU [10]. The purpose of this study was to evaluate the clinical characteristics of patients who underwent surgery for the treatment of leiomyoma after HIFU.

\section{Materials and methods}

We retrospectively reviewed the medical records of 291 patients with leiomyoma who underwent myomectomy or hysterectomy at our institution between June 2016 and September 2017. A total of 12 patients who underwent HIFU to treat leiomyoma prior to an operative procedure were included. Patients that had not undergone pelvic magnetic resonance imaging (MRI) or pelvic sonography were excluded owing to the lack of data for comparing the size of the leiomyoma.

All HIFU procedures were performed at a single local obstetrics and gynecologic clinic. The procedures used the Chongqing Haifu JC (Haifu Technology, Chongqing, China) from June 2016 to May 2017, and then the Chongqing Haifu JC200 (Haifu Technology) was used starting in June 2017. All patients underwent pelvic MRI before and after HIFU to confirm the treatment results. Patients were placed in the prone position, and the treatment process was conducted under intravenous conscious sedation with vital sign monitoring. The treatment protocol utilized an ultrasound wave frequency of $600-700 \mathrm{~Hz}$ and a sonification time of $600-700 / \mathrm{sec} / 1$ shot with an average $300 \mathrm{~W}$ of power. If the patients did not undergo an imaging study within 6 months prior to the first visit, an additional imaging study was performed at the institution. Pelvic sonography was performed to compare the size of the leiomyoma in patients who had undergone MRI within 6 months prior to the procedure. Patients received counseling prior to the operation and decided whether to undergo myomectomy or hysterectomy.

We investigated patient age, parity, body mass index, number of leiomyomas, and International Federation of Gynecology and Obstetrics classification. The leiomyoma size was measured before and after HIFU treatment and before the operative procedures. The weight of the mass was evaluated, and the histopathologic findings were assessed. This study was approved by the Institutional Review Board of Hanyang University Hospital (2018-08-005).

\section{Results}

A total of 12 patients were analyzed. The median age was 45 (range, 28-51) years. Seven patients had children, while the other 5 patients had never had children. The median body mass index was 24.9 (range, 18.1-29.2) kg/m² (Table 1). 


\title{
Obstetrics \& Gynecology Science
}

\author{
Vol. 62, No. 4, 2019
}

Half of all patients had more than 2 leiomyomas. The median size of the leiomyoma was 10.1 (range, 7.8-14.0) cm before HIFU treatment, which changed to 8.75 (range, 5.9-14.8) cm after HIFU. Prior to the operation, the mean tumor size was 9.1 (range, 5.9-18.0) cm (Table 2). The operations were performed for several reasons. Six patients showed an increase in the size of the leiomyoma, 4 patients complained of persistent symptoms, and 2 patients had a newly developed leiomyoma after HIFU. The maximal diameter of the myoma of the 6 patients was $12.8(9.5-16.2) \mathrm{cm}$ before HIFU and $10.3(6.7-18) \mathrm{cm}$ before surgery. Three of the 4 patients complained of menorrhagia, while 1 had dysmenorrhea. The maximal diameter of the myoma in those 4 patients was 10.9 (9.5-14.3) cm before HIFU and $10.1(7.4-13.5) \mathrm{cm}$ before surgery, which showed no statistically significant difference. The median time interval between HIFU and the operation was 7 (range, 3-32) months. Seven patients were sent to our institution after their first HIFU procedure, 4 patients underwent the procedure twice, and 1 patient underwent the procedure more than 3 times (Table 2). Ten of the 12 patients underwent a laparoscopic operation, and 2 patients underwent laparotomy. Four patients underwent hysterectomy, and 8 patients underwent myomectomy. Among the patients who underwent hysterectomy, 2 patients underwent

Table 1. General characteristics of all patients

\begin{tabular}{lc}
\hline Characteristics & Value \\
\hline Age (yr) & $45(28-51)$ \\
Parity & 5 \\
0 & 2 \\
1 & 5 \\
$\geq 2$ & $24.9(18.1-29.2)$ \\
BMl (kg/m²) & \\
No. of leiomyomas (median) & 6 \\
1 & 2 \\
2 & 4 \\
$\geq 3$ & \\
Type of leiomyomas (FIGO classification) & 1 \\
Submucosal (type 0-2) & 12 \\
Intramural (type 3-4) & 3 \\
Subserosal (type 5-7)
\end{tabular}

Data are shown as median (range).

BMI, body mass index; FIGO, International Federation of Gynecology and Obstetrics. laparoscopically assisted vaginal hysterectomy and 2 underwent total laparoscopic hysterectomy (Table 3). The median serum hemoglobin levels before and after the operation were 13.4 (9.6-14.6) g/dL and 10.1 (7.4-11.9) g/dL, respectively. Among the 8 patients who underwent myomectomy, 2 underwent abdominal myomectomy and 6 underwent

Table 2. Clinical characteristics of leiomyoma and high intensity focused ultrasound

\begin{tabular}{lc}
\hline Characteristics & Value \\
\hline Maximal size of leiomyoma $(\mathrm{cm})$ & $10.1(7.8-14.0)$ \\
Initial & $8.75(5.9-9.0)$ \\
Post HIFU & $9.1(5.9-18)$ \\
Pre-operative & \\
Cause of operation & 7 \\
Growing myoma & 3 \\
Aggravation of symptoms & 2 \\
Newly developed myoma & $7(3-32)$ \\
Median periods between last HIFU and & \\
operation (mon) & \\
Counts of previous HIFU procedure & 7 \\
1 & 4 \\
2 & 1 \\
\hline
\end{tabular}

Data are shown as median (range).

HIFU, high intensity focused ultrasound.

Table 3. The results of surgery

\begin{tabular}{lc}
\hline Characteristics & Value \\
\hline Operative procedure & 2 \\
Myomectomy & 6 \\
Laparoscopic myomectomy & \\
Hysterectomy & 2 \\
LAVH & 2 \\
TLH & $190(150-950)$ \\
Weight of specimen (g) & $890(320-1,540)$ \\
Myomectomy & \\
Hysterectomy & 11 \\
Histopathologic report & 1 \\
Leiomyoma & \\
Atypical leiomyoma &
\end{tabular}

Data are shown as median (range).

LAVH, laparoscopically assisted vaginal hysterectomy; TLH, total laparoscopic hysterectomy. 


\section{Obstetrics \& Gynecology Science}

Yun Seo Choe, et al. Optimal condition of surgery after HIFU

laparoscopic myomectomy. The median weight of the tumor was 190 (range, 150-950) g in the myomectomies and 890 (range, 320-1,540) $\mathrm{g}$ in the hysterectomies. The final histopathologic reports indicated that 11 tumors were leiomyomas and 1 was an atypical leiomyoma (Table 3). The most common characteristic of the histopathologic findings was infarction-type necrosis surrounded by granulation tissue with infiltration of lymphocytes and macrophages (Fig. 1).

\section{Discussion}

In this study, although the median leiomyoma size was reduced from $10.1 \mathrm{~cm}$ to $8.75 \mathrm{~cm}$ after HIFU, it increased to $9.1 \mathrm{~cm}$ before the operation. In addition, half of the patients had more than 2 fibroids, and 5 patients received at least 2 HIFU treatments.

A previous study reported that the uterine leiomyoma volume reduction rates after HIFU were $58.08 \%, 66.18 \%$, and $77.59 \%$ at the 3-, 6-, and 12-month follow-up time points, respectively. In addition, the Uterine Fibroid Symptom and Quality of Life rates increased to $42.66 \%, 43.50 \%$, and $43.45 \%$ at the 3-, 6-, and 12-month follow-ups, respectively. Among the 272 patients with uterine leiomyoma that underwent HIFU, 6 experienced symptom recurrence and 1 with cervical leiomyoma underwent a second HIFU treat- ment. Two patients underwent hysterectomy, and 3 patients underwent myomectomy [11]. In other studies, the uterine leiomyoma volume reduction rates after HIFU ranged from $17.2 \%$ to $58.1 \%$ and symptom improvement ranged from $48.2 \%$ to $71.1 \%[4,5,9,11]$. These results demonstrate that HIFU is an effective and noninvasive modality for the treatment of leiomyoma, with a short recovery period. While the effects of HIFU have been studied, its safety has not yet been confirmed $[6,9]$. Moreover, severe adverse events have been reported, including skin burns, bowel perforation, bladder injury, and deep vein thrombosis, all of which required surgical repair $[7,12]$. In particular, studies on fertility and pregnancy outcomes after HIFU are scarce $[5,11]$. Therefore, KSOG has recommended avoiding HIFU in patients who are planning for future pregnancy [8].

In the present study, 6 of the total patients showed an increased leiomyoma size, with an average size of more than $12 \mathrm{~cm}$, due to expansion of an untreated lesion, and 4 patients had persistent symptoms. Four patients had more than 3 leiomyomas and half of the total patients had more than 2 leiomyomas. In addition, 5 patients (42\%) were treated with HIFU 2 or more times. The KSOG has indicated that special attention is required in patients with leiomyomas larger than $12 \mathrm{~cm}$, multiple leiomyomas, and an insufficient response to previous HIFU treatment [8].

HIFU can be used with various size and intensity settings,
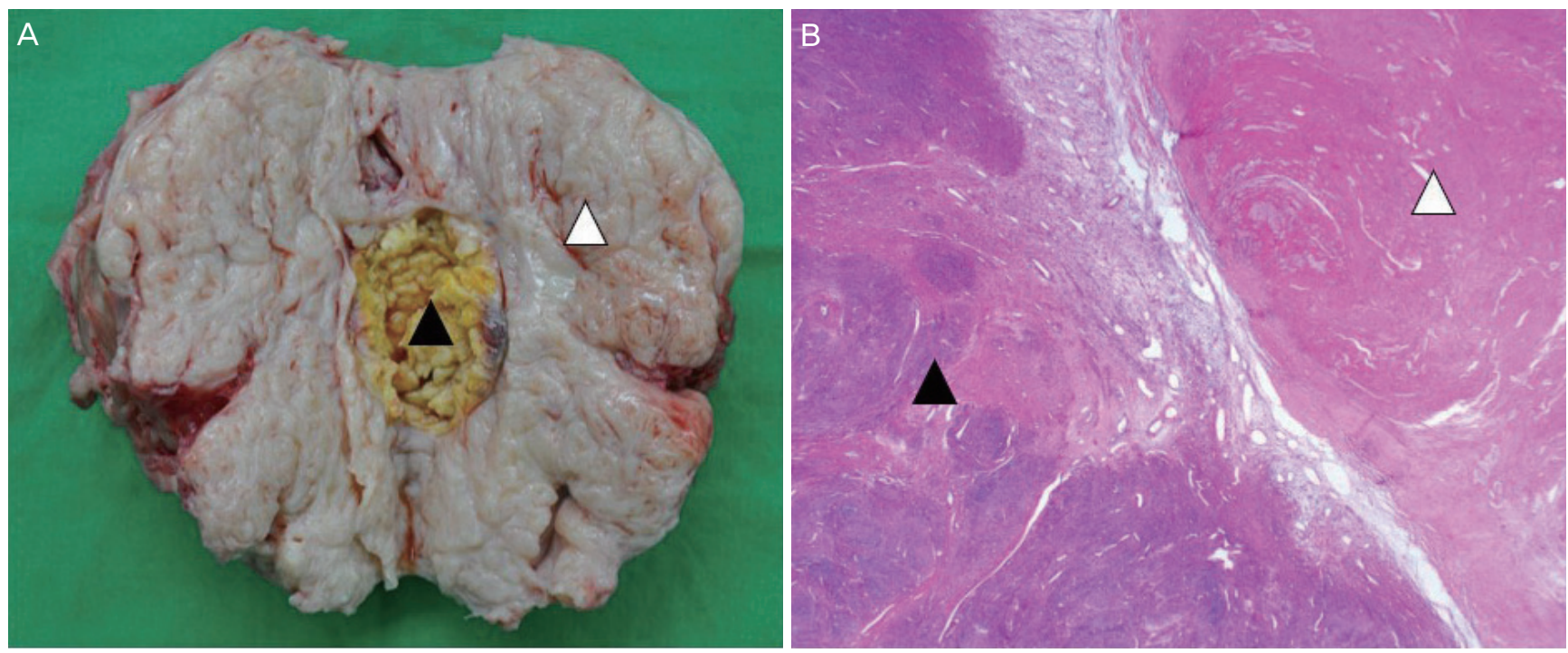

Fig. 1. (A) Post-operative gross appearance of leiomyoma after high intensity focused ultrasound (HIFU). (B) Histopathologic appearance of leiomyoma after HIFU (hematoxylin and eosin stain, $\times 100$ ). Black triangle: leiomyoma with HIFU treatment, white triangle: leiomyoma without HIFU treatment. 


\section{Obstetrics \& Gynecology Science}

Vol. 62, No. 4, 2019

but it is generally a time-consuming treatment. Although the operative time differs according to the energy absorption rate and location of the leiomyoma, it takes an average of 3 hours to treat an 8-cm-sized leiomyoma. Therefore, leiomyomas larger than $10 \mathrm{~cm}$ require multiple HIFU treatments, which can lead to high rates of treatment failure and adverse effects [13]. In a recent study of 36 patients with uterine leiomyomas larger than 10 (range, 10.0-14.3) cm, the HIFU treatment time was reduced to less than 3 hours owing to technological improvements. However, there were still limitations to the size of the leiomyoma that could be treated in that time [14].

As the number of leiomyomas increases, it is likely that at least 1 or more will not be suitable for treatment with HIFU, thereby increasing the possibility of treatment failure. For example, some leiomyomas cannot be treated effectively because they lie outside the transducer range [13]. Additionally, leiomyomas that are close to the sacrum cannot be treated with HIFU because bones absorb ultrasound waves more easily than soft tissue and low energy waves are sufficient to heat the bones to high enough temperatures to cause nerve injury [13]. Furthermore, hypervascular leiomyomas are difficult to treat because the blood vessels steal heat away from the treatment area, resulting in subtherapeutic temperatures $[13,15]$.

There were several limitations to this study. First, it was a retrospective study, and a selection bias may have been introduced by including patients that did not have a successful initial HIFU treatment. Second, the number of patients was too small to verify the significance of the findings. Therefore, an additional, large-scale study should be conducted to compare the characteristics, treatment outcomes including pregnancy, and complications between HIFU and surgery groups.

In conclusion, the treatment of leiomyoma with operative procedures should be considered in selected patients with tumor size greater than $10 \mathrm{~cm}$, multiple tumors, and persistent symptoms after HIFU treatment. HIFU should be recommended for patients who meet the appropriate criteria. Specifically, in patients who show an increased lesion size after HIFU, a second HIFU may be ineffective and operative procedures should be prioritized.

\section{Acknowledgements}

We are grateful to the local gynecology clinic that performed high intensity focused ultrasound and the patients who participated in this study.

\section{Conflict of interest}

No potential conflict of interest relevant to this article was reported.

\section{Ethical approval}

The study was approved by the Institutional Review Board of Hanyang University Hospital (IRB No. 2018-08-005) and performed in accordance with the principles of the Declaration of Helsinki.

\section{Patient consent}

The patients provided written informed consent for the publication and the use of their images.

\section{References}

1. Kim SH, Park HM. Uterine leiomyoma. In: Korean Society of Obstetrics and Gynecology, editor. Obstetrics \& Gynecology. 3rd ed. Seoul: Koonja; 2014. p.77-84.

2. Vilos GA, Allaire C, Laberge PY, Leyland N; SPECIAL CONTRIBUTORS. The management of uterine leiomyomas. J Obstet Gynaecol Can 2015;37:157-78.

3. Duhan N. Current and emerging treatments for uterine myoma - an update. Int J Womens Health 2011;3:23141.

4. Cheung VY. Sonographically guided high-intensity focused ultrasound for the management of uterine fibroids. J Ultrasound Med 2013;32:1353-8.

5. Mahmoud MZ, Alkhorayef M, Alzimami KS, Aljuhani MS, Sulieman A. High-intensity focused ultrasound (HIFU) in uterine fibroid treatment: review study. Pol J Radiol 2014;79:384-90. 


\section{Obstetrics \& Gynecology Science}

Yun Seo Choe, et al. Optimal condition of surgery after HIFU

6. Zhang L, Zhang W, Orsi F, Chen W, Wang Z. Ultrasoundguided high intensity focused ultrasound for the treatment of gynaecological diseases: a review of safety and efficacy. Int J Hyperthermia 2015;31:280-4.

7. Łoziński T, Filipowska J, Gurynowicz G, Gabriel I, Czekierdowski A. Non-invasive therapeutic use of high-intensity focused ultrasound (HIFU) with 3 tesla magnetic resonance imaging in women with symptomatic uterine fibroids. Ginekol Pol 2017;88:497-503.

8. Korean Society of Obstetrics and Gynecology. HIFU guidelines [Internet]. Seoul: Korean Society of Obstetrics and Gynecology; c2016 [cited 2016 Jul 15]. Available from: http://www.ksog.org/bbs/?code=notice\&mode=vi ew\&number $=3525$.

9. Leung JH, Yu SC, Cheung EC, Wong AS, Tong MM, Ho SS, et al. Safety and efficacy of sonographically guided high-intensity focused ultrasound for symptomatic uterine fibroids. J Ultrasound Med 2014;33:1811-8.

10. Sandberg EM, Tummers FH, Cohen SL, van den Haak L, Dekkers OM, Jansen FW. Reintervention risk and quality of life outcomes after uterine-sparing interventions for fibroids: a systematic review and meta-analysis. Fertil Steril 2018;109:698-707.e1.
11. Lee JS, Hong GY, Park BJ, Kim TE. Ultrasound-guided high-intensity focused ultrasound treatment for uterine fibroid \& adenomyosis: a single center experience from the Republic of Korea. Ultrason Sonochem 2015;27:6827.

12. Park J, Lee JS, Cho JH, Kim S. Effects of high-intensityfocused ultrasound treatment on benign uterine tumor. J Korean Med Sci 2016;31:1279-83.

13. Yoon SW, Lee C, Cha SH, Yu JS, Na YJ, Kim KA, et al. Patient selection guidelines in MR-guided focused ultrasound surgery of uterine fibroids: a pictorial guide to relevant findings in screening pelvic MRI. Eur Radiol 2008; 18:2997-3006.

14. Hou R, Wang L, Li S, Rong F, Wang Y, Qin X, et al. Pilot study: safety and effectiveness of simple ultrasoundguided high-intensity focused ultrasound ablating uterine leiomyoma with a diameter greater than $10 \mathrm{~cm}$. Br J Radiol 2018;91:20160950.

15. Kim YS, Kim JH, Rhim H, Lim HK, Keserci B, Bae DS, et al. Volumetric MR-guided high-intensity focused ultrasound ablation with a one-layer strategy to treat large uterine fibroids: initial clinical outcomes. Radiology 2012;263:600-9. 